\title{
Micromapping of very rare anemias: the model of CDA
}

\author{
Hermann Heimpel \\ Department Internal Medicine III, University Hospital of UIm, UIm, Germany
}

\begin{abstract}
The congenital dyserythropoietic anemias, first described in 1966 and classified into four distinct types in 1968, are still very rare. However, many cases were described in recent years, mainly in European Countries. The detection of mutations of the CDAN1-gene (Tamary et al. from Israel) in all cases of CDA I and of the $S E C 23 B$-gene in almost all cases of CDA II (Schwarz, Iolascon, Heimpel et al. from Germany and Italy, Zanella, Bianchi et al. from Italy) stimulated greater awarness of the CDAs and resulted in many recent reports from all continents. Period prevalance of the the last 50 years in the European countries collated in the German Registry on CDAs were calculated in 2009 and published in 2010. The cumulated incidence of both types combined variedwidely between European regions, with minimal values of 0.08 cases/million in Scandinavia and 2.60 cases/million in Italy. CDA II is more frequent than CDA I, with an overall ratio of approximately 3.2 , but the ratio also var-
\end{abstract}

ied between different regions. The most likely explanations for the differences are both differences in the availability of advanced diagnostic procedures and different levels of the awarenessfor the diagnosis of the CDAs. The estimations reported here are most probably below the true incidence rates, because of failure to make the correct diagnosis and to underreporting. Limited data did not suggest differing levels of risk in identified ethnic groups. Here, we report the first results of an update of these data and decribe a project to extent epidemiological research to all regions of the world. Based on the experience with the previous attempt to measure, performed in the framework of ENERCA, we discuss the limitations and methodological problems and present the hypothesis, that the CDAs are not dependent from environmental conditions such as malaria or other contagious diseases, but the apparent prevalence data depend rather on factors of the medical system and the rates of consanguinity in different cultures. Since the prognosis of the CDAs depend on appropriate management, consultation by specialized centers of rare diseases, today available in Europe, could improve life expectancy and quality of life of individuals affected by this very rare diseases.

\author{
Correspondence: Hermann Heimpel \\ (C) Copyright H. Heimpel, 2013 \\ Licensee PAGEPress, Italy \\ Thalassemia Reports 2013; 3(s1):e34 \\ doi:10.4081/thal.2013.s1.e34
}

This article is distributed under the terms of the Creative Commons Attribution Noncommercial License (by-nc 3.0) which permits any noncommercial use, distribution, and reproduction in any medium, provided the original author(s) and source are credited.

Parts of this work were presented at the

"3rd Pan-European Conference on Haemoglobinopathies and Rare Anaemias", Limassol (Cyprus), 24-26 October 2012. 